\title{
Construir ante la adversidad
}

\section{Building in the face of Adversity}

\author{
Carmen Ramírez-Maestre
}

Facultad de Psicología, Universidad de Málaga, España

Disponible online 31 de diciembre de 2016

Numerosas investigaciones empíricas y teóricas, se han centrado en el estudio del bienestar en un intento por comprender los factores implicados en la felicidad humana. Generalmente, el bienestar se ha entendido como la evaluación positiva y subjetiva del individuo sobre su vida (Ryff \& Singer, 2000). La evidencia empírica muestra los múltiples beneficios que supone para la población presentar niveles altos de bienestar (Diener, 2010; Hone, Jarden, Schofield, \& Duncan, 2014), así como los riesgos asociados a bajos niveles de satisfacción (Keyes, 2005, 2010). Es por ello importante trabajar desde la Psicología en el estudio de las variables que facilitan la consecución de satisfacción vital, afecto positivo y, en definitiva, una evaluación subjetiva de bienestar individual y colectivo.

Sin embargo, atendiendo a la literatura científica, el bienestar subjetivo solo hace referencia al bienestar hedónico. De igual (o quizás mayor) importancia es el bienestar psicológico o eudemónico (Ryan \& Deci, 2001). Mientras la perspectiva hedónica alude a "sentirse bien" (SchotanusDijkstra et al., 2016), desde la perspectiva del eudemonismo, el bienestar se refiere al funcionamiento psicológico positivo. Ciertamente, la investigación de los últimos años evidencia un nexo claro entre hedonismo y eudemonismo, de forma que el buen funcionamiento psicológico permitirá niveles deseables de satisfacción y afecto positivo.

En este contexto, la revista Escritos de Psicología publica un número monográfico en el que se recogen trabajos teóricos y empíricos que abordan el estudio del bienestar humano, psicológico y subjetivo, desde diferentes perspectivas y abordajes metodológicos. Dicho número monográfico ha sido coordinado por la autora de este editorial y por los profesores Alfredo Fierro Bardají y Margarita Ortiz-Tallo Alarcón.
Comienza el volumen con dos trabajos teóricos relativos a dos grandes constructos del bienestar psicológico: Fortalezas (Peterson y Seligman, 2004) y Resiliencia (Richardson, 2002).

Desde la Universidad Complutense de Madrid y la Universidad de Málaga, Mercedes Ovejero Bruna, Violeta Cardenal Hernáez y Margarita Ortiz-Tallo nos presentan una revisión sistemática cuyo objetivo es analizar la conexión entre las fortalezas, el bienestar, la felicidad, la satisfacción vital, la resiliencia y la salud mental. Los resultados muestran una clara relación entre el bienestar psicológico (fortalezas) y el bienestar subjetivo operacionalizado como satisfacción vital o felicidad. De esta forma, por ejemplo, son varios los trabajos empíricos que muestran que la vitalidad, el amor, la esperanza y la gratitud son importantes predictores del bienestar, de la satisfacción vital y de la felicidad. De la revisión sistemática, las autoras concluyen además que la identificación y potenciación de fortalezas como la gratitud, la bondad, el trabajo en equipo y el humor se asocia con menores tasas de depresión, afecto negativo y psicopatología, funcionando asimismo como amortiguadoras de los sucesos vitales de impacto negativo, convirtiéndose en importantes indicadores de un desarrollo saludable a largo plazo. De hecho, recogen trabajos que señalan que estas fortalezas podrían ayudar a construir la resiliencia (Martínez -Marti y Ruch, 2016). En este sentido, Peterson et al., (2006) defienden que las fortalezas generan recursos que pueden fomentar la resistencia ante la adversidad e incluso un afrontamiento adaptativo ante la enfermedad física. Entramos así en el contexto del siguiente trabajo: Pain Resilience: Issues of Modeling Dynamic Adaptation in Chronic Pain. John A Sturgeon, de la Universidad de Arizona, nos ofrece un interesante análisis teórico acerca de las distintas concepciones 
de resiliencia, y de su papel en la adaptación al dolor crónico. $\mathrm{El}$ autor concluye que la resiliencia es un constructo complejo, que habría de ser definido como un proceso dinámico que está en constante evolución, de acuerdo con la interacción de las características personales del individuo y los factores situacionales. De esta forma, los pacientes que muestran altos niveles de resiliencia ante el dolor serán capaces de identificar maneras eficaces de afrontamiento que les permitan un mayor bienestar a corto y largo plazo.

De nuevo se analizan las fortalezas en el trabajo Fortalezas psicológicas y diferencias de sexo en adolescentes. Fortalezas de Trascendencia, Felicidad y Salud Mental que nos presentan Sara Reyes Martín y Marta Ferragut. En este caso, hablamos de un estudio empírico cuyo objetivo es examinar el perfil descriptivo de los valores y las fortalezas en una muestra de estudiantes de bachillerato, considerando las diferencias de sexo y ofreciendo un análisis de las fortalezas que componen el valor de trascendencia (apreciación de la belleza, gratitud, optimismo, humor y espiritualidad). Se analiza igualmente la relación entre dichas fortalezas, la felicidad y la salud mental en los adolescentes. Los resultados muestran correlaciones positivas moderadas entre todas las fortalezas del valor trascendencia con la felicidad y la salud mental. La autora concluye que las fortalezas son recursos psicológicos que favorecen el bienestar subjetivo, encontrando algunas diferencias entre hombres y mujeres.

La población joven sigue siendo el foco de atención del trabajo Recursos que contribuyen al desarrollo positivo en jóvenes presentado por Martha Frias Armenta y Melanie Itsel Barrios Gaxiola, de la Universidad de Sonora, México. El objetivo de este estudio fue analizar los recursos que promueven el desarrollo positivo de los jóvenes. Se estudia el desarrollo positivo desde un punto de vista interdisciplinar, sin embargo, las únicas relaciones directas que aparecen en los datos ocurren entre los recursos sociales y psicológicos y el desarrollo positivo del joven. Los resultados apoyan el efecto positivo de la autoestima, la identidad positiva y los valores positivos como la integridad, la responsabilidad, la honestidad, el compromiso social y la justicia en el desarrollo positivo del individuo.

Si bien la mayoría de los trabajos sobre bienestar se han centrado en la población general, la profesora Lourdes Rey, en su trabajo Efecto mediacional del afecto entre las autoevaluaciones centrales y la satisfacción vital en discapacidad intelectual, nos presenta un estudio muy interesante acerca del bienestar subjetivo en una muestra de individuos con discapacidad intelectual. Este estudio incluye un constructo que ha generado un amplio campo de investigación en población normal y que, según la autora, podría tener una potencial influencia sobre el bienestar subjetivo de las personas con discapacidad intelectual: las core self-evaluations (He, Shi y Yi, 2014) o autoevaluaciones centrales. Este concepto representa las evaluaciones fundamentales que los individuos realizan sobre sí mismos, el mundo y los otros. El presente artículo examina el efecto mediacional del afecto en la relación entre las autoevaluaciones centrales y la satisfacción vital, en personas con discapacidad intelectual. La autora señala que altos niveles de autoevaluaciones centrales, a través del afecto positivo, se asocian con una mayor satisfacción con la vida. Por tanto, una formación orientada a aumentar los recursos personales, podría aumentar las experiencias emocionales positivas lo que redundaría en una mayor satisfacción con la vida en el colectivo de personas con discapacidad intelectual.

El monográfico que nos ocupa incluye también un estudio cualitativo llevado a cabo por Manuel Moyano, Manuel I. Bermúdez y Antonia Ramírez, en la Universidad de Córdoba. El artículo titulado ¿Psicología positiva para afrontar la radicalización y el terrorismo? Un análisis del discurso de Obama en el Cairo analiza el discurso " $A$ New Beginning", que el presidente Barak Obama llevó a cabo en El Cairo en 2009. Lo autores lo consideran un ejemplo paradigmático de Psicología Positiva aplicado al afrontamiento de situaciones adversas, encontrando en el texto numerosos pasajes concebidos por los investigadores como indicadores de las virtudes y fortalezas formuladas por Peterson y Seligman (2004) desde el marco de la Psicología Positiva.

Se cierra el volumen con un artículo firmado por el doctor Alfredo Fierro, querido y admirado profesor: Cómo afrontar la pérdida con Proust: un apunte de biblioterapia. Señala el autor que durante siglos se ha escrito para el bienestar, la vida feliz y el buen vivir, remontándose a la obra de Séneca y centrándose en los escritos de Proust. La tesis principal del presente escrito es que el profesional de la psicología, de la modificación del comportamiento, necesita una fuente de conocimiento, más allá de la literatura científica, que sea capaz de alentar y de guiar proyectos realistas de cambio de conducta. Concluye el autor que se aprende y se conoce mejor la conducta y la condición humana con dos clases de lecturas: dramaturgos, novelistas y ensayistas al lado de especialistas, de investigadores, de científicos. Durante la lectura de esta reflexión, una de las 24 fortalezas recogidas en los anteriores trabajos se destaca de manera evidente: la sabiduría del autor.

Finalmente, los trabajos recogidos en este monográfico reflejan el interés, aún presente en la psicología, por conocer los caminos que conducen al bienestar. Del conocimiento de los recursos psicológicos que posibilitan una "vida feliz" se derivan las herramientas que el profesional de la psicología habrá de ceder al individuo, con el objetivo de que éste consiga construir una vida satisfactoria, incluso ante la adversidad.

\section{Referencias}

1. Diener E. (1984). Subjective well-being. Psychological Bulletin, 95, 542-575. https://doi.org/10.1037/00332909.95.3.542

2. Diener, E., Wirtz, D., Tov, W., Kim-Prieto, C., Choi, D., Oishi, S. \& Biswas-Diener, R. (2010). New Well-being Measures: Short Scales to Assess Flourishing and Positive and Negative Feelings. Social Indicators Research, 97, 143- 
156. https://doi.org/10.1007/s11205-009-9493-y

3. He, D., Shi, M. \& Yi, F. (2014). The mediating effects of affect and loneliness on the relationship between core self-evaluation and life satisfaction among two groups of Chinese adolescents. Social Indicators Research, 119, 747756. https://doi.org/10.1007/s11205-013-0508-3

4. Hone, L.C., Jarden, A., Schofield, G.M., \& Duncan, S. (2014). Measuring flourishing: The impact of operational definitions on the prevalence of high levels of wellbeing. International Journal of Wellbeing, 4, 62-90. https://doi. org/10.5502/ijw.v4i1.4

5. Keyes, C.L.M. (1998). Social well-being. Social Psychology Quarterly, 61, 121-140. Keyes, C.L.M., Dhingra, S.S., Simoes, E.J. (2010). Change in level of positive mental health as a predictor of future risk of mental illness. American Journal of Public Health, 100, 2366-2371. https://doi. org/10.2307/2787065

6. Keyes, C. L. M. \& Grzywacz, J. G. (2005). Health as a complete state: the added value in work performance and healthcare costs. Journal of Occupational and Environmental Medicine, 47, 523-532. https://doi.org/10.1097/01. jom.0000161737.21198.3a

7. Martínez-Martí, M. L., \& Ruch, W. (2016). Character strengths predict resilience over and above positive affect, self-efficacy, optimism, social support, self-esteem, and life satisfaction. The Journal of Positive Psychology, 12, 110119. https://doi.org/10.1080/17439760.2016.1163403

8. Peterson, C. \& Seligman, M. E. P. (2004). Character strengths and virtues. A handbook and classification. Nueva York: Oxford University Press.
9. Peterson, C., Park, N., \& Seligman, M. E. P. (2006). Greater strengths of character and recovery from illness. Journal of Positive Psychology, 1, 17-26. https://doi. org/10.1080/17439760500372739

10. Richardson, G. E. (2002). The meta-theory of resilience and resiliency. Journal of Clinical Psychology, 58, 307-321. https://doi.org/10.1002/jclp.10020

11. Ryan, R. M., \& Deci, E. L. (2001). To be happy or to be self-fulfilled: A review of research on hedonic and eudaimonic well-being. In S. Fiske (Ed.), Annual review of psychology (Vol. 52, pp. 141-166). Palo Alto, CA: Annual Reviews/Inc.

12. Ryff, C.D. (1989). Happiness is everything, or is it? Explorations on the meaning of psychological well-being. Journal of Personality and Social Psychology, 57, 1069-1081. https://doi.org/10.1037/0022-3514.57.6.1069

13. Ryff, C. D., \& Singer, B. H. (2000). Interpersonal flourishing: A positive health agenda for the new millennium. Personality and Social Psychology Review, 4(1 Special Issue: Personality and Social Psychology at the Interface: New Directions for Interdisciplinary Research), 30-44. https:// doi.org/10.1207/S15327957PSPR0401_4

14. Schotanus-Dijkstra, M., Klooster, P.M., Drossaert, C.H.C., Pieterse, M.E., Bolier, L., Walburg, J.A. \& Bohlmeijer, E.T. (2016). Validation of the Flourishing Scale in a sample of people with suboptimal levels of mental well-being. BMC Psychology, 4, 12. https://doi.org/10.1186/s40359-016$\underline{0116-5}$ 